\title{
Using Shading and a 3D Template to Reconstruct Complex Surface Deformations
}

\author{
Mathias Gallardo \\ Mathias.Gallardo@gmail.com \\ Toby Collins \\ Toby.Collins@gmail.com \\ Adrien Bartoli \\ Adrien.Bartoli@gmail.com
}

ISIT, UMR 6284 CNRS
Université d'Auvergne
Clermont-Ferrand, France

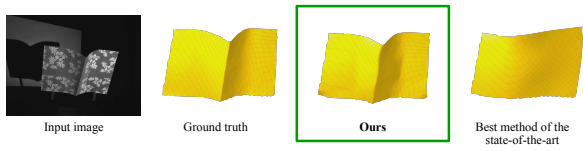

Motivations The goal of Shape-fromTemplate (SfT) is to register and reconstruct the $3 \mathrm{D}$ shape of a deforming surface from a single image and a known deformable 3D template. Most SfT methods use only motion information and require well-textured surfaces which deform smoothly. Consequently they are unsuccessful for poorly-textured surfaces with complex deformations such as creases. However, Shape-from-Shading methods permit to reconstruct textureless surfaces and complex deformations since it uses all image pixels and the photometric relationship. We overcome the shortcomings of previous attempts by proposing a novel, $(i)$ fully-integrated approach to combine shading constraints with SfT in order to (ii) reconstruct complex deformations on all visible regions, both textured and textureless, (iii) without any a priori photometric calibration.

\section{Template, illumination and camera modeling} We define the template as a texture-mapped thin shell 3D mesh in a known reference pose with $M$ vertices. At each time $t$, each vertex is deformed into the unknowns $3 \mathrm{D}$ camera coordinates $\mathbf{x}_{t} \in \mathbb{R}^{3 \times M}$. We upgrade the template with an photometric texture map which defines how each point of the template's surface reflects light. We assume Lambertian model and compute this map using an intensity-based segmentation of the texture-map. It gives constant albedo regions with $\alpha=\left\{\alpha_{1}, \ldots, \alpha_{K}\right\}$, the $K$ unknown albedo values. The scene is illuminated by an unknown illumination $\mathbf{l}$ which is constant over time, fixed in the camera coordinates and modeled by spherical harmonics (4 and 9 coefficients). The camera has a linear response, $\beta_{t} \in \mathbb{R}^{+}$, which is unknown and time-varying.

Integrated cost function The deformation $\mathbf{x}_{t}$ is constrained by image data and deformation priors (isometry and smoothing constraints), and
Figure 1: 3D renderings for the input image $n^{\circ} 6$ of the floral plane dataset.

l, $\beta_{t}$ and $\alpha$ are constrained by the shading term and the batch of images. We use the shading relationship to enforce similarity between the modeled and the measured pixel intensities. As it uses all image pixels, mis-alignement may induce errors. Thus, we use motion and boundary constraints to align the projected 3D surface with its input image. We also use a robust smoothing based on an M-estimator, which permits piecewise constant $3 \mathrm{D}$ reconstructions, such as creases.

Strategy solution The integrated cost function is large scale and highly non-linear, but all constraints are sparse with respect to $\mathbf{x}_{t}$. We use a cascaded initialization for the four types of unknowns: first $\mathbf{x}_{t}$, then using a batch of input images $\mathbf{l}, \beta_{t}$ and finally $\alpha$. Using Gauss-Newton iterations with line-search, a refinement process minimizes the whole integrated cost function for the batch of images. We found that a dense mesh with vertices of order $\mathcal{O}\left(10^{4}\right)$ is sufficient to capture the creases.

Experimental results We compare our approach on three datasets with four SfT methods and we see that our method is capable of capturing non-smooth deformations, better than others, as figure 1 shows, using shading without any a priori photometric calibration, which was not possible with previous methods in SfT or SfS. 